\title{
Effects of task outcome and subjective standard on state depression for cognitive and social tasks
}

\author{
LOUIS S. DICKSTEIN and ANNE WHITAKER \\ Wellesley College, Wellesley, Massachusetts 02181
}

\begin{abstract}
Experimental studies of depression in college students have often focused on task outcome. The present study explores the role of subjective standard as a cognitive mediator of the effects of success and failure on mood for both cognitive and social tasks. Subjective standard is defined as the lowest score with which a subject would be satisfied. The data showed a significant main effect of task outcome, a significant main effect of subjective standard, and a significant interaction between these variables on both depression scores and a measure of subject satisfaction with her score. The findings are interpreted as providing support for a cognitive model of depression.
\end{abstract}

In recent years, much research has been conducted on depression among college students. This research has been prompted by reports that depression is common on the college campus, and these reports are supported by empirical studies. Thus, Bosse, Croghan, Greenstein, Katz, Oliver, Powell, and Smith (1975) found that 75\% of college sophomores at Washington University asked to complete the Beck Depression Inventory retrospectively reported having experienced at least mild depression during the preceding year, and $45 \%$ of the respondents reported moderate or severe depression. More recently, Oliver and Burkham (1979) found that $17 \%$ of undergraduate and graduate students at St. Louis University were at least mildly depressed with depression rates higher for underclassmen.

One of the most commonly investigated variables in experimental studies of depression in college students has been task outcome. These studies have compared success outcomes with failure outcomes and have found that subjects report more depression after failure experiences. Most of these studies have utilized cognitive tasks. Thus, Flippo and Lewinsohn (1971) used a puzzle solving task and reported a significant effect of task outcome on self-esteem. Klein, Fencil-Morse, and Seligman (1976) utilized a learned helplessness paradigm in which discrimination learning problems were either solvable or insolvable and found that subjects in the solvable group were significantly lower in sadness self-ratings than were subjects in the insolvable and control groups. Fry (1976) employed three different tasks (perceptual motor skill, recall recognition, and analogies) over three separate sessions and then obtained self-assessments on 10 social psychological items. Subjects in the success group had significantly higher self-assessments on positive items

The research reported in this paper was conducted by the second author in partial fulfillment of the requirements for honors in psychology, under the supervision of the first author. than did failure and control subjects, whereas subjects in the failure group had significantly higher self-assessments on negative items than did success and control subjects. Furthermore, these effects persisted $24 \mathrm{~h}$ after completion of the tasks. Finally, Golin, Jarrett, Stewart, and Drayton (1980) used an anagram task and demonstrated a main effect of a success-failure manipulation on depression scores on the Multiple Affect Adjective Check List.

Although cognitive tasks have been most common in research on task outcome, some studies have employed tasks that were presented as measures of social skills. Thus, Wener and Rehm (1975) used an association task that was described to subjects as a measure of social intelligence and interpersonal empathy. Similarly, Hammen and Krantz (1976) gave their subjects a tape recording of a client expressing concerns to a therapist, and the subjects were asked to select the proper therapeutic responses to these concerns. Task outcomes purportedly reflected subjects' social competencies. In these studies, the effects of task outcome have been more equivocal. Thus, Wener and Rehm (1975) failed to obtain a main effect on depression when subjects receiving $80 \%$ reinforcement were contrasted with subjects receiving $20 \%$ reinforcement, although a reanalysis of the data in which some subjects were dropped did yield a significant difference. Similarly, Hammen and Krantz (1976) failed to obtain significant differences in self-ratings between success, failure, and no-feedback groups among nondepressed subjects, although there was a significant effect of task outcome for depressed subjects.

It is difficult to draw any firm conclusions regarding possible differences between cognitive and social tasks on the basis of these studies, since they were conducted by different experimenters with different samples using different procedures and measures. One purpose of the present study was to directly compare the effects of success and failure on cognitive and social tasks. 
The effects of success and failure on depressive state are consistent with behavioral models (e.g., Wener \& Rehm, 1975) that view depression as a response to the amount or rate of positive reinforcement provided by the environment. These results, however, are not inconsistent with cognitive models (e.g., Beck, 1974) that maintain that the way an individual appraises events determines whether the events will induce depression (see Blaney, 1977; Lobitz \& Post, 1979). The effects of task outcome may be mediated by cognitive variables.

If the effects of task outcome are mediated by cognitive variables, one such variable may be the individual's subjective standard of performance, that is, the level of performance the individual finds satisfactory. Thus, an individual's reaction to a particular task outcome may be a function of the degree of correspondence between actual performance and subjective standard. Subjects with higher subjective standards may be less satisfied with their own performance than subjects with lower subjective standards, and this may be reflected in a higher state of depression for subjects with higher standards. While this relationship may hold for both success and failure outcomes, it appears most likely that there would be a significant interaction between task outcome and subjective standard. Thus, standards may not play a central role when subjects experience success, but they may be very crucial in the evaluation of failure. The negative impact of failure may be much greater for subjects with higher standards than for subjects with lower standards.

Several studies with college students support a relationship between subjective standards and depression. Thus, Nelson (1977) reported a correlation of .46 between depression and high self-expectations as measured by the Irrational Beliefs Test. More depressed subjects expressed a greater need to excel in all endeavors to feel worthwhile. Similarly, Golin and Terrell (1977) found that depressed subjects have higher maximum and minimum goals on a test of information than do nondepressed subjects. Finally, some indirect evidence regarding standards has been reported by Bierman (1981), who found that given the same objective level of performance on a social empathy task, nondepressed subjects are significantly more likely to rate their own performance as successful than depressed subjects are. These differences in evaluation of the same objective performance presumably reflect differences between the groups in subjective standards.

While these studies show that subjective standards are correlated with depressive mood, they do not address the possible role of subjective standard as a mediator of the reactions of subjects to different task outcomes. The present study was designed to explore this issue for both cognitive and social tasks. It is predicted that there will be significant main effects of both task outcome and subjective standard on subject reactions to their performance and subject mood. More important, a significant interaction between task outcome and subjective standard is predicted.

\section{METHOD}

Subjects consisted of 88 female undergraduates who were paid for their voluntary participation. Each subject participated in an individual session with a female experimenter.

The design of the study was a 2 by 2 by 2 factorial with three between-subjects variables: type of task, task outcome, and subjective standard. Type of task was manipulated as an independent variable. Half of the subjects were randomly assigned to a cognitive task group, and half were assigned to a social task group. Subjects in the cognitive group were given an "analytic reasoning test," which consisted of six analogies from a sample Miller Analogies Test and 14 analogies that appeared to have answers but were, in fact, meaningless. The test was in a multiple-choice format with four alternatives per item. Subjects in the social group were given a "social reasoning test," which consisted of 20 statements regarding appropriate behavior in social situations. Although all of the statements were matters of opinion, subjects were told that there were correct answers that had been found to correlate with social adjustment. A truefalse format was used.

Task outcome was also manipulated as an independent variable. Half of the subjects in each task condition were randomly assigned to a success feedback group, and half were assigned to a failure feedback group. The feedback provided consisted of the subject's alleged test score, the alleged average score for students at the institution, and a histogram allegedly depicting the distribution of scores for 200 students. On the basis of a pilot study, it was determined that feedback was most believable for these tasks within a range from $60 \%$ to $90 \%$ correct. Consequently, " $75 \%$ correct" was used as the average score in the "norms" for all subjects in both tasks. Subjects in the success condition were told they had received $90 \%$ correct, and subjects in the failure condition were told they had received $60 \%$ correct. In addition, subjects were told that their scores represented a level either somewhat higher or somewhat lower than average for students at the institution.

Subjective standard was defined as a subject variable and was measured with a pretest questionnaire administered to all subjects immediately following description of the task but prior to performance on the task. This questionnaire contained several questions relating to subjects' perceptions of the task and of their own ability. Subjective standard was defined as the subject's answer to the question, "What is the lowest score you could get and still be satisfied?" In order to obtain an equal number of subjects in each cell for statistical analysis, subjects in each of the four cells defined by type of task and task outcome were divided at the median subjective standard for that cell. Subjects who were tied at the median in any cell were randomly assigned. This resulted in a total of eight different groups with 11 subjects in each group.

After subjects had taken the test, they were invited to look over the "norms" while the experimenter ostensibly scored the test. In order to control for experimenter bias in the administration of the session, the experimenter did not know the subject's feedback condition until this point in the proceedings, when a list was consulted. The subject was then shown her "scored" test and given the appropriate verbal feedback for that condition. Immediately following feedback, the subject was administered the Depression Adjective Check List, Form C (Lubin, $1965)$ as a measure of state depression. Following this measure, subjects were asked to complete a posttest questionnaire soliciting subject perceptions of the task, her score, and her ability. One of the items on this questionnaire was the question, "How do you feel about your score?" followed by three response alternatives: "pleased," "disappointed," and "neither." Responses to this item were scored on a 3-point scale with a 
score of 3 for "pleased" and a score of 1 for "disappointed." Scores on this item and on the Depression Adjective Check List constituted the dependent variables of this study. After completing the posttest questionnaire, subjects were debriefed and desensitized

\section{RESULTS}

Since subjects were randomly assigned to cognitive and social tasks and to success and failure conditions without regard to subjective standard, a preliminary twoway analysis of variance was conducted to check that the four groups defined by these variables did not differ in subjective standard. Neither the main effects nor the interaction was significant.

Means and standard deviations for the question, "How do you feel about your score?" for all groups are presented in Table 1. Differences between the groups were assessed with a three-way analysis of variance. As expected, there was a significant main effect of task outcome $[F(1,80)=104.72, p<.001]$. The mean for the success condition was 2.73 ; the mean for the failure condition was 1.64. Also, as expected, there was a significant main effect of subjective standard $[F(1,80)=$ $6.56, \mathrm{p}<.05]$. The mean for low subjective standard subjects was 2.32 ; that for high subjective standard subjects was 2.05 . In addition, as expected, there was a significant interaction between task outcome and subjective standard $[\mathrm{F}(1,80)=6.52, \mathrm{p}<.05]$. Thus, in the success condition, there was no difference between the means for high and low subjective standard subjects. Both means were 2.73. However, in the failure condition, there was a substantial difference between the groups, with a mean of 1.91 for low subjective standard subjects and a mean of 1.36 for high subjective standard subjects. Finally, there was an unexpected significant main effect of type of task $[F(1,80)=4.56, p<.05]$. The mean for the cognitive task condition was 2.30; that for the social task condition was 2.07 .

Means and standard deviations for the Depression Adjective Check List for all groups are presented in Table 2. Differences between the groups were assessed with a three-way analysis of variance. As expected, there was a significant main effect of task outcome $[F(1,80)=$ $14.75, \mathrm{p}<.001]$. The mean for the failure condition was 10.89; the mean for the success condition was 6.84. Also, as expected, there was a significant main effect of subjective standard $[F(1,80)=7.39, p<.01]$.

Table 1

Means and Standard Deviations on Reaction-to-Score Measure

\begin{tabular}{|c|c|c|c|c|c|c|c|c|}
\hline \multirow[b]{3}{*}{ SS } & \multicolumn{4}{|c|}{ Cognitive } & \multicolumn{4}{|c|}{ Social } \\
\hline & \multicolumn{2}{|c|}{ Success } & \multicolumn{2}{|c|}{ Failure } & \multicolumn{2}{|c|}{ Success } & \multicolumn{2}{|c|}{ Failure } \\
\hline & Mean & SD & Mean & SD & Mean & SD & Mean & SD \\
\hline High & 2.73 & .47 & 1.55 & .69 & 2.73 & .47 & 1.18 & .40 \\
\hline Low & 2.91 & .30 & 2.00 & .63 & 2.55 & .52 & 1.82 & .40 \\
\hline
\end{tabular}

Note-SS $=$ subjective standard.
Table 2

Means and Standard Deviations on Depression Adjective Check List

\begin{tabular}{|c|c|c|c|c|c|c|c|c|}
\hline \multirow[b]{3}{*}{ SS } & \multicolumn{4}{|c|}{ Cognitive } & \multicolumn{4}{|c|}{ Social } \\
\hline & \multicolumn{2}{|c|}{ Success } & \multicolumn{2}{|c|}{ Failure } & \multicolumn{2}{|c|}{ Success } & \multicolumn{2}{|c|}{ Failure } \\
\hline & Mean & SD & Mean & SD & Mean & SD & Mean & SD \\
\hline High & 7.18 & 5.91 & 13.82 & 5.91 & 7.27 & 7.51 & 12.91 & 4.21 \\
\hline Low & 6.45 & 3.14 & 10.09 & 2.51 & 6.45 & 4.99 & 6.73 & 3.20 \\
\hline
\end{tabular}

Note-SS = subjective standard.

The mean for high subjective standard subjects was 10.30 ; that for low subjective standard subjects was 7.43. Finally, the interaction between task outcome and subjective standard fell just short of statistical significance $[F(1,80)=3.94, p<.10]$. The critical value needed for statistical significance at the .05 level is 3.98. There was no main effect of type of task, and none of the other interactions approached significance.

Since there was no significant effect of type of task and none of the interactions with type of task even approached significance, it was decided to combine the data from the cognitive and social tasks and conduct a two-way analysis of variance for task outcome and subjective standard. This procedure increases the power of the statistical test for an interaction. Again, there was a significant main effect of task outcome $[F(1,84)=$ $14.98, \mathrm{p}<.001]$ and a significant main effect of subjective standards $[F(1,84)=7.50, p<.01]$. The interaction was also significant $[F(1,84)=4.00, p<.05]$. In the success condition, there was a small difference between the means for high and low subjective standard subjects ( 7.23 vs. 6.45$)$, but in the failure condition, there was a much larger difference between high and low subjective standard subjects (13.36 vs. 8.41).

As a supplement to the main analysis, additional three-way analyses of variance were conducted on other questions from the pretest questionnaire. On the pretest, there were two significant differences between high and low subjective standard subjects. On the question "How certain are you of your analytic/social reasoning ability?", high subjective standard subjects expressed significantly more confidence than low subjective standard subjects $[F(1,80)=4.89, p<.05]$. Similarly, on the question "How important to you is doing well on this task?", high subjective standard subjects expressed greater concern about their performance than low subjective standard subjects $[\mathrm{F}(1,80)=5.37, \mathrm{p}<.025]$. There were no main effects on the pretest for type of task or task outcome, and none of the interactions approached significance.

\section{DISCUSSION}

The results of this study indicate that task outcome, subjective standards, and the interaction between them are all important factors in subjects' reactions to their own performance on both cognitive and social tasks. This is the case for both dependent variables utilized in this study. However, the pattern of the data is slightly different in the two instances. 
When subjects are asked specifically about their reactions to their own scores, there is a clear main effect of task outcome. Subjects in the success condition are pleased with their scores, whereas subjects in the failure condition are disappointed with their scores. This difference is significant for both high and low subjective standard subjects. Although there is also a significant main effect of subjective standard, this main effect is qualified by the interaction. In the failure condition, subjects with low subjective standards are significantly less disappointed with their performance than subjects with high subjective standards. However, in the success condition, both high and low subjective standard subjects are equally satisfied with their performance.

On the more general measure of depressive mood, both main effects of task outcome and subjective standard are qualified by the interaction. There is a significant effect of task outcome for high subjective standard subjects, but not for low subjective standard subjects. Similarly, there is a significant difference between high and low subjective standard subjects in the failure condition, but not in the success condition. Another way of describing these findings is that the mean depression score for high subjective standard subjects in the failure condition is significantly higher than the means in the other three cells, which, in turn, are not significantly different from each other. In fact, the mean for the high subjective standard subjects in the failure condition of 13.36 approaches the norm for female depressed psychiatric patients of 15.71 reported by Lubin (1967), whereas the other means are all close to the norm of 7.87 reported for female college students.

The difference in the pattern of results for the two dependent variables indicates that while task outcome has a direct effect upon subject satisfaction with her score, the effect of task outcome on depressive mood is mediated by the subjective standards subjects bring to the task. When such standards are high, task outcome will have a much stronger impact than when such standards are low. These findings are consistent with the cognitive mediation model of depression.

It is the contention of this paper that subjective standards constitute an antecedent cognitive variable that mediates between task outcome and depressive mood. However, since subjective standard was measured as a subject variable, it is possible to maintain that the results simply reflect a positive correlation between subjective standards and depression. Subjects higher in subjective standard may have entered the study with higher levels of depression. While the design of this study does not allow this alternative interpretation to be definitively rejected, it is important to note that such an interpretation appears incompatible with a supplementary finding. As reported earlier, there was a main effect of subjective standard on the pretest question, "How certain are you of your analytic/social reasoning ability?" Subjects with high subjective standards expressed significantly more confidence than subjects with low subjective standards. Since Fibel and Hale (1978) have reported that depression in college students is negatively correlated with expectancy for success, whereas Golin and Terrell (1977) have reported no difference between depressed and nondepressed subjects in task expectancy, it appears unlikely that higher levels of self-confidence would be expressed by more depressed subjects. Such a finding would also run counter to studies that have consistently found depressed subjects to be lower in selfesteem (Flippo \& Lewinsohn, 1971). In the light of this finding, the cognitive mediation explanation appears more plausible.

The results of the present study suggest that the effects of task outcome and subjective standard hold across both cognitive and social tasks as defined in this study. Although there was an unexpected main effect of task type on satisfaction with score, there were no significant interactions between task type and the other variables. Furthermore, neither the main effect nor the interactions involving task type were significant for the depression measure.
The present study demonstrates that task outcome and subjective standards play an important role in the depressive mood of female undergraduates. Further research is necessary to determine whether similar findings would be obtained with male subjects. In addition, more information is needed about the generality and stability of subjective standards. For example, do subjects with high subjective standards on a cognitive task also have high subjective standards on a social task? Again, how stable are subjective standards over the course of a student's college career? Finally, research is needed on the antecedents of individual differences in subjective standards. More information regarding these issues may help in finding ways to reduce the incidence of depression among college students.

\section{REFERENCES}

BEck, A. The development of depression: A cognitive model. In R. Friedman \& M. Katz (Eds.), The psychology of depression: Contemporary theory and research. Washington, D.C: Winston, 1974.

Bie rman, K. Relationship between stability of cognition and stability of depressive mood. Psychological Reports, 1981, 48, 295-298.

Blaney, P. Contemporary theories of depression: Critique and comparison. Journal of Abnormal Psychology, 1977, 86, 203223.

Bosse, J., Croghan, L., Greenstein, N., Katz, N., Oliver, J., Powell, D., Smith, W. Frequency of depression in the freshman year. Journal of Consulting and Clinical Psychology, $1975,43,746-747$.

Fibel, B., \& Hale, W. The Generalized Expectancy for Success Scale: A new measure. Journal of Consulting and Clinical Psychology, 1978, 46, 924-931.

Flippo, J., \& Lewinsorn, P. Effects of failure on the self esteem of depressed and non-depressed subjects. Journal of Consulting and Clinical Psychology, 1971, 36, 151.

FRY, P. Success, failure and self assessment ratings. Journal of Consulting and Clinical Psychology, 1976, 44, 413-419.

Golin, S., Jarrett, S., Stewart, M., \& Drayton, W. Cognitive theory and the generality of pessimism among depressed persons. Journal of Abnormal Psychology, 1980, 89, 101-104.

Golin, S., \& TERrell, F. Motivational and associative aspects of mild depression in skill and chance tasks. Journal of Abnormal Psychology, 1977, 86, 389-401.

Hammen, C., \& Krantz, S. Effect of success and failure on depressive cognitions. Journal of Abnormal Psychology, 1976, 85, 577-586.

Klein, D., Fencil-Morse, E., \& Seligman, M. Learned helplessness, depression and the attribution of failure. Journal of Personality and Social Psychology, 1976, 33, 508-516.

Lobitz, W., \& Post, R. Parameters of self reinforcement and depression. Journal of Abnormal Psychology, 1979, 88, 33-41.

LuBin, B. Adjective check lists for the measurement of depression. Archives of General Psychiatry, 1965, 12, 57-62.

Lubin, B. The depression adjective check list. San Diego: Educational and Industrial Testing Service, 1967.

NeLson, R. Irrational beliefs in depression. Journal of Consulting and Clinical Psychology, 1977, 45, 1190-1191.

Oliver, J., \& BurkhaM, R. Depression in university students: Duration, relation to calendar time, prevalence, and demographic correlates. Journal of Abnormal Psychology, 1979, 88, 667-670.

Wener, A., \& Rehm, L. Depressive affect: A test of behavioral hypotheses. Journal of Abnormal Psychology, 1975, 84, 221-227.

(Received for publication February 7, 1983.) 